\title{
A influência das ferramentas big data e inteligência artificial no marketing 4.0
}

\author{
The influence of big data and artificial intelligence tools in marketing 4.0 \\ La influencia de las herramientas de big data e inteligencia artificial en el marketing 4.0
}

Recebido: 21/04/2021 | Revisado: 29/04/2021 | Aceito: 30/04/2021 | Publicado: 07/05/2021

\author{
Danihanne Borges e Silva \\ ORCID: https://orcid.org/0000-0002-9627-2929 \\ Instituto Federal de Educação, Ciência e Tecnologia Goiano, Brasil \\ E-mail: sublimefestas2017@gmail.com \\ Daniela Cabral de Oliveira \\ ORCID: https://orcid.org/0000-0002-9647-933X \\ Instituto Federal de Educação, Ciência e Tecnologia Goiano, Brasil \\ E-mail: danielacaboliveira@gmail.com \\ Dilça Cabral de Jesus \\ ORCID: https://orcid.org/0000-0002-8557-0429 \\ Universidade de Rio Verde, Brasil \\ E-mail: dilcac@gmail.com
}

\begin{abstract}
Resumo
A adequação da inteligência artificial e da big data no marketing 4.0 tornaram a abordagem do marketing inovadora, contribuindo para o aumento das vendas, vantagem competitiva, aumento do ROI, otimização dos processos, personalização avançada para os clientes e entregar experiências inovadoras para os clientes. O objetivo do estudo foi apresentar informações sobre a quarta revolução industrial, o impacto da big data nos negócios, a inteligência artificial e seus subcampos e por fim o marketing 4.0. Nessa revisão qualitativa os artigos, foram selecionados nas bases de dados Scielo e Google Acadêmico, também foram selecionados livros para o desenvolvimento do trabalho. Como resultado final foi dado ênfase sobre como as ferramentas de inteligência artificial e big data revolucionaram o marketing.
\end{abstract}

Palavras-chave: Inteligência artificial; Big data; Marketing.

\begin{abstract}
The suitability of artificial intelligence and big data in marketing 4.0 made the approach to marketing innovative, contributing to increased sales, competitive advantage, increased ROI, process optimization, advanced customization for customers and delivering innovative experiences for customers. The objective of the study was to present information about the fourth industrial revolution, the impact of big data on business, artificial intelligence and its subfields and, finally, marketing 4.0. In this qualitative review, the articles were selected from the Scielo and Google Scholar databases, and books were also selected for the development of the work. As a final result, emphasis was placed on how artificial intelligence and big data tools revolutionized marketing.
\end{abstract}

Keywords: Artificial intelligence; Big data; Marketing.

\section{Resumen}

La idoneidad de la inteligencia artificial y el big data en el marketing 4.0 hizo que el enfoque del marketing fuera innovador, contribuyendo al aumento de las ventas, la ventaja competitiva, el aumento del ROI, la optimización de procesos, la personalización avanzada para los clientes y la entrega de experiencias innovadoras para los clientes. El objetivo del estudio fue presentar información sobre la cuarta revolución industrial, el impacto del big data en los negocios, la inteligencia artificial y sus subcampos y, finalmente, el marketing 4.0. En esta revisión cualitativa, los artículos fueron seleccionados de las bases de datos Scielo y Google Scholar, y también se seleccionaron libros para el desarrollo del trabajo. Como resultado final, se hizo hincapié en cómo la inteligencia artificial y las herramientas de big data revolucionaron el marketing.

Palabras clave: Inteligencia artificial; Big data; Marketing.

\section{Introdução}

Com a evolução das tecnologias, os consumidores e as organizações passaram a adotar as mesmas com a finalidade de melhorar suas atividades. E no marketing não foi diferente, as ferramentas de tecnologia alteraram a maneira como é abordado 
o marketing. Nesse sentido o marketing 4.0 tornou uma abordagem inovadora devido também ao grande volume de dados, big data, inteligência artificial e comportamento do consumo no ambiente digital.

No marketing 4.0 os varejistas on-line acompanham não só o que os clientes compram, mas também o que eles mais buscam, como eles navegam através do site, o quanto eles são influenciados pelas promoções, revisões e layouts de páginas (McAfee et al., 2012). De acordo com Kotler e Keller (2013) para alcançar o sucesso, o marketing 4.0 deve ser holístico e construir marcas fortes através de desempenho em vez de promoção. O marketing 4.0 precisam levar eletronicamente, bem como, através da construção de sistemas superiores de informação e comunicação.

McAfee et al., (2012) afirmam que o fluxo constante de informações sobre o público consumidor e suas interações é fundamental para projetar novas práticas de marketing, tais como compra de mídia programática, segmentação de público, gatilho de marketing em tempo real.

Diante desse cenário a ferramenta big data é considerada uma estratégia de marketing. Nesse sentido, o uso de big data no marketing 4.0 tem a função de ser onipresente, universal, original, e, portanto, a marca deve rever conceitos e paradigmas para melhorar a experiência e engajamento dos consumidores (Vargo \& Lush, 2004). E assim a big data fornecem informações preciosas para o marketing 4.0 tornando as marcas sensíveis às demandas do mercado (Donelly et al., 2015).

Outra aplicação no marketing é a inteligência artificial incluindo as implicações da marcação social em conteúdos online no desempenho da marca, neuromarketing, estratégia e política de preços, atendimento ao cliente com chatbots. O marketing tornou-se um beneficiário natural da inteligência artificial, tirando proveito dos dados em seus processos, desde a pesquisa de necessidades do consumidor, análises de mercado, insights de clientes e análise de concorrência, através da realização de atividades em vários canais de comunicação ou distribuição até à medição dos resultados e efeitos das estratégias adotadas (Jarek \& Mazurek, 2019). Neste sentido, é possível afirmar que a inteligência artificial revolucionou o marketing.

\section{Desenvolvimento}

Este trabalho trata-se de uma pesquisa qualitativa, baseada em uma revisão exploratória, conforme metodologia proposta por Gil (2008). Os artigos para compor este trabalho foram selecionados livros, leis e artigos das bases de dados Scielo e Google Acadêmico, utilizando as palavras-chave: quarta revolução industrial, big data, inteligência artificial e marketing.

A busca foi realizada, em primeiro momento, de forma rápida e objetiva, com leitura dos títulos dos artigos e excluindo os artigos duplicados disponíveis em mais de uma base de dados. Em seguida, foi realizada a leitura dos artigos previamente selecionados, os quais incluíram todos os trabalhos que relatassem diretamente quarta revolução industrial, big data, inteligência artificial e marketing. Na sequência, fez-se a ordenação das informações coletadas e realizou-se uma leitura crítica do referencial teórico.

\section{Referencial Teórico}

\subsection{Quarta Revolução Industrial}

Segundo Schwab (2016) afirma que na quarta revolução industrial novas descobertas ocorrem simultaneamente em áreas que vão desde o sequenciamento genético até a nanotecnologia das energias renováveis à computação quântica. $\mathrm{O}$ autor afirma também que a quarta revolução industrial é a fusão dessas tecnologias e a interação entre os domínios físicos, digitais e biológicos.

Segundo Barbosa et al., a quarta revolução industrial é distinta das outras revoluções devido aos seguintes fatores: a velocidade evolui em ritmo exponencial e não linear, a amplitude e a profundidade são marcadas pela revolução digital como 
base, combinando várias tecnologias e o impacto sistêmico transformando os sistemas em organizações, indústrias e em toda sociedade.

Já Castells (1999) afirma que o novo paradigma dessa revolução informacional é que a informação é sua matériaprima: são tecnologias para agir sobre a informação, não apenas informação para agir sobre tecnologia, como foi o caso das revoluções anteriores.

Diante desse contexto, Schwab (2016) afirma que a quarta revolução industrial:

Cria uma unidade de riqueza com muito menos trabalhadores, em comparação há 10 ou 15 anos, porque os custos marginais das organizações digitais tendem a zero. Além disso, na realidade da era digital, muitas novas organizações oferecem "bens de informação" com custos praticamente nulos de armazenamento, de transporte e de replicação. Organizações como o Instagram ou o Whatsapp, por exemplo, não exigem muito financiamento para iniciar, mudando o papel do capital e a escala dos negócios no contexto da quarta revolução industrial.

Schwab (2016) aponta alguns critérios que diferenciam a quarta revolução industrial das anteriores: velocidade, amplitude e impacto sistêmico. Segundo o autor, a quarta revolução industrial evolui exponencialmente, enquanto as outras Revoluções se desenvolveram de forma linear. A revolução digital serve de base de apoio, uma vez que, para o surgimento das inovações tecnológicas, é necessário um grande poder computacional para analisar big data, criar algoritmos e inteligência artificial.

Schwab (2016) afirma que a inteligência artificial (IA) está em nosso entorno, em carros que pilotam sozinhos, drones, assistentes virtuais e softwares de tradução. A IA fez progressos impressionantes, impulsionada pelo aumento exponencial da capacidade de processamento e pela disponibilidade de grandes quantidades de dados, desde softwares usados para descobrir novos medicamentos até algoritmos que preveem nossos interesses culturais. $\mathrm{O}$ autor afirma que os algoritmos de inteligência artificial aprendem a partir de dados que deixamos no mundo digital, resultando em novos tipos de aprendizagem automática e detecção automatizada que possibilitam que robôs inteligentes e computadores a se auto programar e encontrar as melhores soluções a partir de princípios iniciais. Por exemplo, programas como a Siri da Apple, também chamado de assistentes inteligentes, demonstra a progressão da velocidade do reconhecimento de voz e a inteligência artificial criando algo que os especialistas chamam de computação ambiental.

Segundo Schwab (2016) a quarta revolução industrial tornou possível a existência de novos produtos e serviços que aumentam, sem praticamente nenhum custo, a eficiência de nossas vidas como consumidores. Como por exemplo, solicitar um táxi, encontrar um voo, comprar produtos, realizar pagamentos, ou seja, qualquer uma dessas tarefas pode ser realizada à distância. E nesse sentido, é possível afirmar que a internet, o smartphone e os aplicativos estão deixando nossas vidas fáceis e produtivas.

Costa e Pagani (2019) afirmam que no relatório Artificial Intelligence, Automation, Economy a mudança tecnológica recente tende a afetar a força de trabalho de uma forma diferente do que ocorreu no século XIX, aumentando a produtividade relativa dos trabalhadores qualificados, eliminando ou diminuindo a demanda por ocupações que exijam trabalhos repetitivos ou tarefas rotineiras.

De acordo com Schwab (2016) afirma que a quarta revolução industrial em sua iniciativa na transformação digital da indústria destaca uma série de negócios e modelos de funcionamento com foco no cliente. Por exemplo, a Nespresso, concentram seus esforços nos processos de linha de frente e capacitam seus funcionários para que coloquem o cliente em primeiro lugar. $\mathrm{O}$ autor também afirma que os modelos de negócios criam novas fontes de receitas a partir do acesso de informações valiosas sobre os clientes. Por exemplo, a Skynet concentra na automação, tornando-se mais prevalente em locais e indústrias perigosas. Na área automotiva, a eletrônica representa aproximadamente $40 \%$ do custo do carro. A Apple e a 
Google entraram no mercado automotivo e isso retrata que uma organização de tecnologia pode transformar em uma organização automobilística.

\subsection{Big Data}

Big Data são dados transacionais, armazenados, metadados que residem em arquivos grandes. Segundo Lima Junior (2011), a big data se refere ao conjunto de dados, cujo volume está além dos padrões e da capacidade das ferramentas utilizadas por bancos de dados para capturá-los, analisá-los e gerenciá-los.

Mapelli et al. (2018) define a big data como de uso e aplicação a partir de uma análise de dados disponíveis, em base gigantesca, alimentada constantemente, em um modelo que se adequa a novos panoramas, sem intervenções humanas. Nesse sentido a autora afirma que a análise de big data gera avanços sociais significativos sendo considerado um instrumento para a real transparência de informação.

Segundo Bulian e Alencar (2017) define:

A big data cria valor para as organizações descobrindo padrões e relacionamentos entre os dados que antes estavam perdidos não apenas em Data Warehouse, Web, Twitter, comentários no Facebook e mesmo em vídeos no YouTube. A big data extrai novas ideias e cria novas formas de valor alterando mercados, organizações, relação entre cidadãos e governos. Esta mudança quantitativa advinda de big data tem gerado mudança qualitativa em termos de resultados do processamento de dados.

Mayer-Schonberger e Cukier (2014) afirmam que a big data não é somente sobre tamanho, mas especialmente sobre a habilidade de transformar em dados muitos aspectos do mundo que nunca foram quantificados antes.

Segundo Mendes e Mattiuzzo (2019) afirmam que:

A função mais importante da big data é elaborar previsões baseadas em grandes números de dados e informações: desde desastres climáticos até crises econômicas, do surto de uma epidemia até o vencedor de um campeonato de esportes, do comportamento de um consumidor até a solvência dos clientes. Assim, as análises de big data podem ser utilizadas para elaborar prognósticos, tanto com relação à economia, à natureza ou à política, quanto sobre comportamento individual.

Mapelli et al. (2018) afirmam que em 2009 se pôde com a aplicação algorítmica em big data, antecipar o surto da gripe H1N1 em diversas regiões do mundo. Outros exemplos de seus usos podem ser vistos na prevenção de crimes ou de desastres naturais. Ou seja, a big data é uma ferramenta, e sua análise tem e terá aplicações positivas, ou negativas, de acordo com o uso.

Nesse contexto, surge a análise de big data também chamada de big data analytics, ou seja, análise de uma grande quantidade de dados utilizando tecnologias, processos e práticas que permitem as organizações analisar dados, orientando as tomadas de decisões e o gerenciamento das atividades de forma eficiente.

Gandomi e Haider (2015) definem analytics como técnicas utilizadas para analisar e obter informação inteligente a partir de uma big data. Os autores também afirmam que a big data analytics pode ser visto como um subprocesso que faz parte do processo geral de extração do conhecimento da big data.

Já Kaplan e Norton (2004) descrevem que aplicações analíticas em um ambiente de negócios são sistemas e redes que promovem a analise, a interpretação e o compartilhamento de informações e conhecimento.

A análise de dados corresponde ao conjunto de atividades desempenhadas, desde a seleção dos dados até a produção do conhecimento, envolvendo a busca de padrões consistentes, detectando relacionamentos sistemáticos entre as variáveis e gerando conhecimento não detectado. As atividades que compõem o processo de análise de dados são: seleção, pré- 
processamento, métodos de análise e avaliação. A análise de dados é apoiada por conjunto de métodos que incluem métodos de mineração de dados pré-processamento, classificação, predição, agrupamento, associação e visualização. Esses métodos são apoiados por técnicas como: K-means, Partitioning Around Medoids (PAM), Árvores de decisão, Redes neurais, Support Vector Machines (SVM).

\subsubsection{Aplicação da Big Data}

A Big Data em serviços representa as seguintes dimensões: novo conceito de serviços; nova interação com o cliente; novo modelo de receitas e novo sistema de entrega: tecnologia. O Linkedin, por exemplo, estabelece um conceito de serviço, ou seja, funcionalidade PQTVC. O PQTVC sugere aos clientes do Linkedin conectar com outros usuários. As agências de viagens Decolar e Kayak oferecem ofertas desejáveis aos clientes, baseada em consultas de usuários e preços atualizados de companhias aéreas. A Netflix oferece conceitos de serviço: assistir filmes online baseado na tecnologia streaming como nova forma de entregar o produto para o cliente. Outro exemplo é o aplicativo EasyTaxi com função localizar e solicitar um táxi pelo celular. O aplicativo identifica as localizações do usuário e dos taxistas próximos através do GPS do celular. O aplicativo permite uma nova forma de comunicação entre taxistas e clientes, que não modifica o serviço em si, mas agrega comodidade, rapidez e segurança. A indústria petrolífera incorporou dados meteorológicos e preços dos concorrentes para determinar os preços dos produtos e/ou serviços.

De acordo com Schwab (2016) para melhor informar os frequentadores do restaurante sobre locais insalubres, São Francisco, em colaboração com o Yelp, montou um programa piloto de êxito, fundindo os dados das inspeções sanitárias feitas pela cidade nos restaurantes às páginas de avaliação do site. O autor também afirma que desde Iowa até a Índia, os agricultores estão usando os dados de sementes, satélites, sensores e tratores para melhorar as decisões sobre o que plantar, quando plantar, como monitorar o frescor dos alimentos da fazendo à mesa e como se adaptar às mudanças climáticas.

\subsection{Inteligência Artificial}

Segundo Hoffman-Riem (2019) a inteligência artificial é uma tecnologia transversal que visa capacitar computadores, mediante a utilização de computadores, mediante a utilização de big data, capacidades computacionais apropriadas e processos específicos de análise e decisão, a alcançar realizações que se aproximam da capacidade humana.

Já de acordo com Madeira et al. (2020) a inteligência artificial é ainda mais complexa. Existem diferentes abordagens, a partir do pensamento e comportamento, humanos ou não. Essas abordagens perseguem a similaridade ao desempenho humano ou a um modelo ideal de inteligência, a dita racionalidade. Russel e Norvig (2013) enquadra a inteligência artificial em quatro grupos - pensando como humano, pensando racionalmente, agindo como humano e agindo racionalmente.

Segundo Artero (2009) as principais áreas de atuação da inteligência artificial são: robótica: dispositivos robóticos têm se tornado comum na indústria, sendo capazes de realizar tarefas que exigem força e precisão; Visão por computador: tem como objetivo desenvolver a capacidade de extrair informações do ambiente a partir de imagens obtidas; Processamento de linguagem natural: tradução online de conversas entre pessoas de diferentes etnias; Sistemas especialistas: sistemas que conseguem fornecer respostas, sobre um determinado domínio, a um usuário; Reconhecimento de padrões: reconhecer faces, gestos, palavras escritas e faladas e o próprio ambiente; Base de dados inteligentes: habilidade de raciocinar, de modo que seja gera resultados novos e; Prova de teoremas: solução de problemas em diversas áreas, em se definir sequências lógicas de ações que levam de uma situação inicial (hipótese) até o objetivo final (tese).

Madeira et al. (2020) o aprendizado de máquina é um subcampo da inteligência artificial e diz respeito a sistemas autodidatas que acessam grandes quantidades de dados, analisam-nos e aprendem a partir deles, sem que tenham sido explicitamente programados para tal. Existem sistemas de aprendizado de máquina para negócios desenvolvidos por 
organizações como Amazon, Google e Baidu. Os autores afirmam também que para a inteligência artificial o conceito de agente é uma característica interessante dos ambientes de compra online, pois os vendedores criam interfaces de venda com feições interativas.

Russell e Norvig (2013) a inteligência artificial é um campo de estudos da computação que se dedica ao estudo e desenvolvimento de sistemas que possam reproduzir comportamentos inteligentes e cumprir tarefas complicadas com um grau de confiabilidade que é equivalente ou superior ao de um humano.

A inteligência artificial está presente em corretores automáticos de texto em computadores, tablets e smartphones a diagnósticos médicos baseados em leitura de imagem, atendimentos a clientes, treinamento de funcionários e gestão de fortunas através de plataformas como Watson da IBM, um sistema que fala e entende o que as pessoas falam. O sistema aprende com a própria experiência, oferecendo resultados cada vez mais inteligentes (Vortigo, 2017).

Nesse sentido surge o conceito de chatbots. Segundo Shiraishi et al., (2020, p. 20) afirmam que o chatbot oferece o toque humano na interface digital, proporcionando conversas e interações entre humano e máquina similares às interações entre humanos em um ambiente digital, altamente e com baixa presença humana". Já Bohle (2018) afirma que um chatbot é um programa de computador projetado para interagir com as pessoas emulando de perto a conversação humana, como idealizado no Teste de Turing para inteligência artificial.

Russell e Norvig (2013) abrangem a inteligência artificial numa variedade de subcampos, desde a aprendizagem e percepção até tarefas específicas, como jogos de xadrez, demonstração de teoremas matemáticos, criação de poesias, direção de carro, diagnóstico de doenças e marketing.

Segundo Madeira et al. (2020) a inteligência artificial e seus impactos no marketing e suas consequências são muito diversificados. Por meio dos seus processos complementares, como o aprendizado de máquina, big data e internet das coisas, a inteligência artificial fez a sua presença ser sentida e produndamente ser estudada. Usar a inteligência artificial para uma melhor experiência do usuário consumidor, análises preditivas e marketing direcionado, fornecendo um grande ROI para os negócios.

\subsection{Marketing 4.0}

O conceito de marketing se fundamenta na satisfação de desejos do usuário-consumidor/cliente e envolve iniciativas que atuam desde o planejamento e a criação de produtos até estratégias de vendas, passando por intervenções nos processos de produção. Iniciativas que envolvem pesquisas de mercado, design de produto, campanha publicitária, promoção de pós-venda, etc (Kotler, Kartajaya \& Setiawan, 2017).

E diante da junção do marketing com a tecnologia surge o marketing 4.0. Kotler (2016) afirma que o marketing 4.0 está centrado no humano, com objetivo dos consumidores interagindo com os produtos, com conversações entre pessoas conduzidas em uma voz humana, com proposição de valor e personalidade, com campos humanitários e conversas pessoais com os clientes. O autor define que o marketing 4.0 está centrado na área das aspirações humanas, valores e espírito. Ele propõe que os consumidores são seres humanos completos cujas outras necessidades e esperanças nunca devem ser negligenciadas. Já Bismas e Roy (2015) definem que o marketing 4.0 integra o consumidor com a tecnologia e também fornece soluções baseadas na análise da informação dos consumidores sobre suas preferências, opiniões e necessidades. Enfim, o marketing trabalha as mudanças observadas no comportamento de consumo em decorrência da popularização dos serviços digitais, da interação entre os canais de comunicação e do crescimento de consumo de conteúdo da web.

Segundo Turban et al., (2015) todo fluxo de informações aumentam o poder de estratégia da organização, neste sentido o marketing utiliza a big data para conhecer, entender e mensurar as necessidades e comportamentos dos novos 
consumidores com intuito de melhorar a relação com os consumidores. Já Hoffman et al., (2010) afirmam que a big data transforma os negócios tradicionais em oportunidades para a vantagem competitiva. Os gestores realizam previsões e decisões inteligentes, bem como alvo de intervenções eficazes em áreas que têm sido especuladas por instinto e intuição do que dados e rigor. Kotler (2016) afirma que a big data apoia e reforça o modelo do marketing 4.0, usando os dados adquiridos de compra dos clientes faz com que as organizações entendam como aprimorar a experiência de compras do cliente e como realizar uma abordagem mais personalizada de marketing da marca levando a um melhor envolvimento do cliente, retenção e fidelização, e otimização de marketing e desempenho. De acordo com um estudo de Harvard sobre big data, as organizações que adotam Web Analytics conseguem ser 5\% mais produtivas e são 6\% mais previsíveis quando tomam decisões baseadas em dados.

Nesse sentido, Dunn (2015), destaca que o marketing 4.0 transforma as regras de negócio, alterando a interação com os clientes no tempo e no espaço. Sashi (2012) afirma que o marketing está descrito em termos de engajamento da marca, fidelização, retenção e recomendação. Kurkovsky e Harihar (2006) afirmam que as vendas on-line são consolidadas, de modo que as estratégias de marketing começam a ser projetadas de acordo com as lojas do navegador web. Wortmann \& Fluchter (2015) a evolução da tecnologia vai além interação humano-computador. Já Vassileva (2017) afirma que as organizações podem acessar grandes quantidades de informação sobre as relações marca-consumidor através da multiplicidade de canais que influenciam o processo de tomada de decisão.

Outra área que tende a contribuir muito para o marketing 4.0 é a inteligência artificial. Segundo Madura (2008) um relatório da Salesforce publicado em 2017, apresenta uma detalhada fotografia de tendências e insights em marketing, mostrando como as novas tecnologias estão sendo encaradas e absorvidas dentro das organizações. Foram mais de 3.500 profissionais pesquisados, incluindo o Brasil. $\mathrm{O}$ estudo conclui que inteligência artificial é a tecnologia com maior crescimento de uso em marketing nas organizações, estimando um crescimento de $53 \%$ na adoção de inteligência artificial, seguido pela internet das coisas. Segundo o documento, os profissionais de marketing afirmam que inteligência artificial ajuda o marketing em 3 frentes: permitir campanhas eficazes, assegurar o melhor ROI, gestão dos assets, otimização dos processos em marketing, entre outros; oferecer uma personalização avançada para os clientes e entregar experiências inovadoras para os clientes.

Segundo Madura (2008) 57\% dos profissionais de marketing avaliam que a inteligência artificial tem um impacto substancialmente a automação das interações através de chatbots e outras interfaces com o cliente. Ou seja, já existe uma consciência de que inteligência artificial realmente leva a relação com o cliente a um outro patamar.

\section{Considerações Finais}

O marketing 4.0 é considerado um novo contexto de negócios transformado pela incorporação da tecnologia na vida das pessoas. O marketing 4.0 encoraja as organizações a procurar objetivos com base no comprometimento dos consumidores. E nesse sentido, a big data ajuda o marketing a alcanças as metas das organizações, compreendendo o engajamento dos consumidores e, assim, projetar ações de marketing personalizadas baseadas nas experiências de compra do cliente.

Já a inteligência artificial aplicada ao marketing 4.0 dá espaço a inovação baseado nos dados coletados, na pesquisa do consumidor na internet, comportamento de compras personalizando assim a experiência do consumidor a um novo patamar. Um exemplo de aplicação da inteligência artificial no marketing 4.0 são os chatbots. Os chatbots interagem com os consumidores com base nos dados que recebe e interfere na experiência do usuário-cliente.

\section{Referências}

Artero, A. O. (2009). Inteligência artificial teórica e prática. 45. Livraria da Física.

Barbosa, J. S., Silva, D. B., Oliveira, D. C. \& Jesus, D. C. (2021). A proteção de dados e segurança da informação na pandemia COVID-19: contexto nacional, 10(2), e 40510212557, Research, Society and Development. 
Research, Society and Development, v. 10, n. 5, e50210515296, 2021

(CC BY 4.0) | ISSN 2525-3409 | DOI: http://dx.doi.org/10.33448/rsd-v10i5.15296

Bismas, A., \& Roy, M. (2015). Green products: Na exploratory study on the consumer behaviour in emerging economies of the East. Journal of Cleaner Production, 87, 463-468.

Bohle, S. (2018). "Plutchnik": artificial intelligence chatbot for searching NCBIdatabases. Journal of the Medical LibraryAssociation: JMLA, 106(4).

Bulian, B. S. \& Alencar, C. A. (2021). A importância da big data para a compreensão dos hábitos de compra dos consumidores, aumentando as vantagens competitivas no comércio varejista. https://www.nucleodoconhecimento.com.br/administracao/big-data-comercio-varejista.

Castells, M. (2005). A sociedade em rede: a era da informação - economia, sociedade e cultura. 1(8), Paz e Terra.

Costa. M. N. A. \& Pagani, M. (2019). Quarta Revolução Industrial e o Futuro do Trabalho. https://juslaboris.tst.jus.br/bitstream/handle /20.500.12178/167897/2019_costa_marcelo_4a_revolucao.pdf?sequence=1\&isAllowed=y.

Donnelly, C., Simmons, G., Armstrong, G., \& Fearne, A. (2015). Digital loyalty card 'big data' and small business marketing: Formal versus informal or complementary? Intenational Small Business Journal, 33(4), 422-442.

Dunn, L. (2015). Globalization and consumer:What marketer needs to know. The Neumann Business Review, 16-30.

Gandomi, A. \& Haider, M. (2015. Beyond the hype: Big data concepts, methods, and analytics. International Journal of Information Management. 35(2), 137144.

Gil, A. C. (2008). Métodos e Técnicas de Pesquisa Social. (6a ed.), Atlas.

Hoffman, D. L., Praveen, K. K., \& Thomas, P. N. (2010). The Right Consumers for Better Concepts: Identifying and Using Consumers High in Emergent Nature to Further Develop New Product Concepts, Journal of Marketing Research.

Jarek, K., \& Mazurek, G. (2019). Marketing and Artificial Intelligence. Central European Business Review, 8(2), 46-55. https://doi.org/10.18267/j.cebr.213

Kaplan, R. \& Norton, D.P. (2004). Mapas estratégicos - Balance Scorecard: convertendo ativos intangíveis em resultados tangíveis. Elsevier.

Kotler, P. (2016). Marketing 4.0 - Moving from traditional to digital. Hoboken (Livro eletrônico). John Wiley, Inc.

Kotler, P., Kartajaya, H. \& Setiawan, I. (2017). Marketing 4.0: moving from traditional to digital. Ed Wiley.

Kotler, P., \& Keller, K. L. (2013). A framework for marketing managementPrentice Hall.

Kurkovsky, S., \& Harihar, K. (2006). Using ubiquitous computing in interactive mobile marketing. Personal and Ubiquitous Computing, 10(4),227-240.

Madeira, A. C. M., Neves, B. C. \& Branco, D. J. B. C. (2020). O Uso da Inteligência Artificial Aplicada ao Marketing Digital: Exploração das Vulnerabilidades do Usuário-Consumidor. Journal of Digital Media and Interaction. 3(8). 95-111.

Mapelli, A.; Giongo, M. \& Carnevale, R. (2018). Os impactos das novas tecnologias do direito e na sociedade. 233. Editora: Deviant Ltda. Erechim.

Mayer-Schonberger, V. \& Cukier, K. (2014). Big data: a revolution that will transform how we live, work, and think. first mariner books.

McAfee, A, Brynjolfsson, E., Davenport, T. H., Patil, D. J., \& Barton, D. (2012). Big data. The management revolution. Harvard Bus Ver, 90(10), 61-67.

Mendes, L. S. \& Mattiuzzo, M. (2019). Discriminação algorítmica: conceito, fundamento legal e tipologia. 16(90), 39-64. Revista Direito Público. Porto Alegre.

Maduro, S. (2018). Inteligência Artificial Aplicada a Negócios. Revista Inteligência Competitiva. 8(3).

Russell, S. \& Norvig, P. (2013). Inteligência Artificial. Tradução de Regina de Regina Célia Simile. (3a ed.),, Elsevier.

Sashi, C. M. (2012). Customer engagement, buyer-seller relationship, and social media. Management Decision, 50(2), 253-272.

Schwab, K. (2016). A Quarta Revolução Industrial. Editora Edipro. Edições Profissionais Ltda.

Schwab, K. (2019). Aplicando a Quarta Revolução Industrial. Prefácios de Satya Nadella, João Doria; Tradução: Daniel Moreira Miranda. Edipro, Título original: Shaping the fourt Industrial Revolution.

Shiraishi, G. de F.; Yoda, F. S. \& Lourenço, V. C. (2020). Para o high-tech ser high touch - um estudo exploratório com chatbots, 22(1), Rio de Janeiro.

Transformação Digital. Página Inicial. (2018). <http://transformacao. digital/>.

Turben, E., King, D., Lee, J. K., Liang, T. P, \& Turban, D. C. (2015). Overview of Eletronic Commerce. In Eletronic Commerce 3-49. Springer International Publishing.

Vargo, S. L., \& Lusch, R. F. (2004). Evolving to a New Dominant Logic for Marketing. Journal of Marketing, 68(1), 1-17.

Vassileva, B. (2017). Marketing 4.0: How Technologies transform marketing organization. Obuda University e-Bulletin, 7(1), 47.

Vortigo. (2017). 5 aplicações de inteligência artificial nas empresas. http://blog.vortigo.com.br/5-aplicacoes-de-inteligencia-artificial-nas-empresas/.

Wolfgang, H. (2019). Inteligência Artificial Como Oportunidade para a Regulação Jurídica. 16(90). Revista Direito Público. 
Research, Society and Development, v. 10, n. 5, e50210515296, 2021

(CC BY 4.0) | ISSN 2525-3409 | DOI: http://dx.doi.org/10.33448/rsd-v10i5.15296

Wortmann, F., \& Fluchter, K. (2015). Internet of thing. Business \& Information Systems Engineering, 57(3),221-224. 